\title{
MICROHONGOS DEL SUELO Y ASOCIADOS A PASTOS SILVESTRES SENESCENTES EN UNA LOCALIDAD PRECORDILLERANA DE LA $9^{2}$ REGIÓN (CHILE)
}

\author{
Eduardo Piontelli,L \& Maria Angelica Grixolli.A \\ Universidad de Valparaiso, Escuela de Medicina, \\ Cátedra de Micología. Casilla 92 V. Valparaiso (Chile).
}

Palabras clave: Microhongos: suelo, pastos sene-scentes, gramíneas.

Key words: Microfungy, soil, senescent grasses, gramineas.

\section{RESUMEN}

Mediante métodos de dilución y cámara húmeda, se analizó cualitativa y cuantitativamente la micota presente en el suelo y en su cobertura de pastos senescentes (gramineas principalmente), en una localidad precordillerana de pradera en la $9^{a}$ región. Nuestra finalidad fue la obtención de datos preliminares sobre la diversidad y composición de especies en suelos subtropicales.

La diversidad de géneros fue mayor en los pastos (38), que en el suelo(27), mientras el número de especies en ambos habitat fue similar (50 y 48). Solo 14 especies fueron comunes en ambos sustratos (28,9\%), representadas mayoritariamente por Hyphomycetes (71,4\%) y Coelompcetes $(21,45 \%$ ) Los taxa dominantes en los suelos fueron: Cladosporium cladosporioides, Penicillium spp. y $\quad l$ as levaduras blancas. En los pastos senescentes: Acremonium spp. Alternaria alternata, A. tenuissima, Botrytis cinerea, Cladosporium cladosporioides, $C$. herbarum, Dactylaria candidula, Epicoccum purpurascens, Fusarium poae, Gliocladium catenulatum, G. roseum, Heteroconium chaetospira, Leptosphaeria ogilviensis, Myrothecium verrucaria, Papulaspora inmersa, Penicillium spp., Periconia minutissima, Phoma levellei, y Torula herbarum.

En los suelos, a diferencia de los pastos, se presentó una gran diversidad de taxa esporádicos (40).

\section{INTRODUCCION}

Los estudios referentes a los diferentes tipos de comunidades fúngicas presentan ciertas dificultades metodológicas e interpretativas cuando son analizadas

\section{SUMMARY}

[Microfungi of soil and associated to senescent wild grass in a preasedine locality of the IX Region (Chile)]

By means of dilution methods and moist chamber cultures, the mycota present in soil and its coberture of senescent grasses (mainly gramineous), were analyzed quantitatively and qualitatively, in a pre-mountain locality of praires in the Ninth Region.

Our aims was to obtain preliminary data on the variety and composition of species in subtropical soils.

The variety of genera were higher in grasses (39) than in soil (27), while the number of species in both habitat were similar (50 and 48). Only 14 species were common in both substrate $(28,9 \%)$, represented mainly by Hyphomycetes (71,4\%), and Coelomycetes (21,45\%).

The dominant taxa in the soil were: Cladosporium cladosporioides, Penicillium spp and white yeast. In senescent grasses: Acremonium spp, Alternaria alternata, A. tenuissima, Botrytis cinerea, Cladosporium cladosporioides, C.herbarum, Dactylaria candidula, Epicoccum purpurascens, Fusariump poae, Gliocladium catenulatum, G.roseum, Heteroconium chaetospira, Leptosphaeria ogilviensis, Myrothecium verrucaria, Papulospora inmersa, Penicillium spp, Periconia minutissima, Phoma levellei, and Torula herbarum.

In the soils, unlike grasses a great variety of sporadic taxa were present (40).

estructuralmente. Esta situación no puede abordarse sin establecer una adecuada correlacion con ciertos parámetros ambientales tales como, el tipo de vegetación y la composición del suelo, considerados ambos como factores primarios desde los trabajos pioneros de Haas(1932) Leischner-Siska (1939) y Warcup (1951).

El análisis de la micocenosis en diversas partes del mundo, se focaliza ya sea con el empleo de los macrohongos en la caracterización más profunda de la fitocenosis(Bon \& Gehu, 1973, Lange, 1978, Losa Quintana 
et al. 1980), pero también con los microhongos en el estudio de las asociaciones con plantas y animales (Lamb \& Brown. 1970, Wicklow 1973, Hubalek, 1978, Clarke \& Christensen,1981, Rambelli et al. 1983-1984, Widden, 1986).

A zesar de que la información cualitativa obtenida de los microhongos dei suelo o de aquellos capaces de colonizar las estructuras aéreas senescentes, es siempre incompleta cuando es analizada en el laboratorio mediantes métodos directos (cultivos artificiales y ambiente controlado), permite estimar, densidad, fluctuación y frecuencia relativa de las especies en el habitat.

En la literatura nacional existen pocas referencias actuales sobre microhongos(Asco-Deuteromycotina) saprotrofos del suelo y de gramíneas (Tollenaar et al. 1970). Como muchas especies descritas no se han publicado en la literatura. el mayor listado de especies fúngicas aún se condensa en la obra de Mujica y Vergara (1980). Nuevos aportes sobre la biodiversidad fúngica en diversos habitat chilenos, se enumeran en un reciente y aún inédito trabajo del Prof. Waldo Lazo (comunicación personal).

Los principales objetivos de nuestra investigación fueron: 1).-Determinar en un área precordillerana, la variación y la abundancia de especies fúngicas de ambas comunidades, ya sea en el suelo como en su cobertura de pastos siltvestres. 2). En estosúltimos. estudiar las presencia y diversidad de su micota en áreas foliares. inflorescencias y tallos senescentes.

\section{MATERIALES Y METODOS}

\section{Descripción dell área y biogeogråía}

La ubicación geográfica de la zona de muestreo fue la $9^{\circ}$ Región, Latitud $38^{\circ} \mathrm{S}$, a la altura de la ciudad de Victoria, a unos $30 \mathrm{~km}$. hacia el Este, entrando por el camino del pueblo Ingeniero Fernández y cercana al Parque Nacional de Tolhuaca (Fig.1).

La fecha de muestreo fue a principios del mes de Mayo de 1993, en plena estación otoñal. La zona presenta características climáticas transicionales entre la $8^{a}$ y $9^{a}$ Región. es de tipo templado cálido lluvioso (cercano a los $2000 \mathrm{~mm}$ al año), con una altura sobre el nivel del mar de unos $600 \mathrm{~m}$. La presencia de asentamientos urbanos y rurales ha disminuido notoriamente la cobertura boscosa, apareciendo en cambio un paisaje de tipo parque antropofiizado con grandes áreas bajo explotación agropecuaria (Quintanilla,1983), alternadas con la presencia de pequeños bosques o grupos de árboles de una sola especie, generaimente roble o pellin (Notofagess). También se observa la presencia de algunos lingues, avellanos. peumoy radal. Todos los caminos. cercos de los campos y límites de los bosques están rodeados de zarzamora, yaquil o espino amarillo (Ulex egropeus). Sin embargo. la presencia de maqui y quila es también común.
El tipo de terreno es negruzco arcilloso, con un $\mathrm{pH}$ ácido cercano al 6. Carbono total 3,32\%, Caliza activa (óxido de calcio) $2.26 \%$, Nitrógeno $0,022 \%$. El lugar, seleccionado al azar. correspondió a terrenos de llanura cubiertos principalmente de pastizales (algunos árboles autóctonos y matorral bajo), destinados a la engorda y pastoreo de vacunos y caballares.

Fig. 1 Area Geográîca Muestral (x)

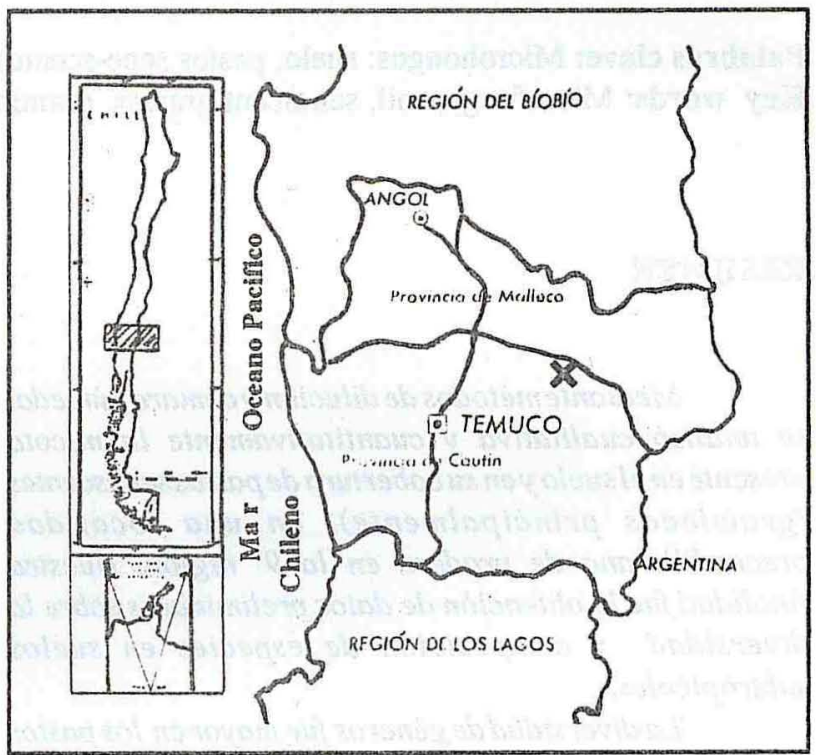

Técmica de muestreo

El sector de muestreo fue una franja de terreno de alrededor de unos 50 metros de ancho, paralelo al camino hacia el Parque Nacional de Tolhuaca, que se extiende de W a E. a lo largo de una distancia de $1.8 \mathrm{Km}$.

El muestreo se efectuó empleando dicho camino como transecto y penetrando cada $100 \mathrm{~m}$. alternadamente a derecha e izquierda unos $20-30 \mathrm{~m}$. en los potreros colindantes. En esa ubicación, se recorría en círculo un área de $50 \mathrm{~m} 2$ app. tomando 7 a 9 submuestras de suelo, que se guardaban en una bolsa plástica estéril, la cual representaba una unidad muestral. El suelo colectado fue aproximadamente de unos 250 g para cada unidad nuestral. obtenido por raspado superficial (hasta 5 cm de profundidad) con una cuchara metálica, esterilizada quinicamente con una solución de yodo all $2 \%$.

Con esta metodología, se obtuvieron 18 unidades muestrales de suelo.

Los pastos se colectaron simultáneamente en cada parcela ( $\mathrm{n}=18$ ) y con el mismo método. empleando un cuchillo metálico y seccionándolos en pequeños manojos a unos $10-15 \mathrm{~cm}$ del suelo, hasta completar la mitad del contenido de una bolsa plástica estéril, de $15 \times 40 \mathrm{~cm}$.de diam. Estos no se seleccionaron ni catalogaron morfológicamente, solo se escogieron al azar los senescentes. los cuales. debido a la época. eran abundantes en todas las 
parcelas de muestreo.

Los gémeros y especies de pastos se determinaron posteriormente en el laboratorio y estaban representados mayoritariamente por especies de: gramineas (Poacege),

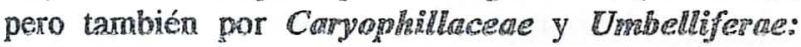

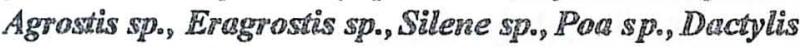
glomercar y Dascus capota. (dominando los 3 últimos). Algunos no pudieron determinarse por la pérdida de sus inflorescencias.

Después de colectadas las muestras se refrigeraron a $3-4{ }^{\text {a}} \mathrm{C}$ durante 48 horas antes de su siembra.

Técricas de siemahra s y cultivos.

Empleamos las técnica de dilución en placa (Warcup, 1950), en el medio PDA con Cloramfenicol $(0,10 \mathrm{~g} / \mathrm{l})$ y Dichloran $(0,0020 \mathrm{~g} / \mathrm{l})$. Este último, para reducir el tamaño de las colonias de los mucorales. El pH final fue regulado en 5,5 .

Para la técnica de dilución, se pesó $1 \mathrm{~g}$ de suelo de cada muestra, se sembraron alicuotas de $0,1 \mathrm{ml}$ de cada dilución $(1 / 10,1 / 100,1 / 1000)$ en 2 series de 3 placas de Petri para cada muestra. Se incubaron por 7 a 30 días a temperatura ambiente de laboratorio $\left(15-20^{\circ} \mathrm{C}\right)$. Las placas se observaron semanalmente, aislándose algunos de los hongos presentes en otros medios selectivos cuando fue necesario (Czapek, MEA, SNA, PCA, u otros).

Para los pastos se empleó la técnica de cámaras húmedas en placas de Petri, sin determinar el tipo de pasto empleado (selección al azar), cortándolos en trozos de 5 $\mathrm{cm}$, hasta cubrir parcialmente el fondo deéstas. Se adicionó agua destilada semanalmente durante 60 días, para mantener una humedad relativa constante $(>$ de $70 \%$ ). Se tuvo especial cuidado en agregar poca cantidad de agua a la vez $(2$ a $3 \mathrm{cc}$ ), para no inundar el fondo de las placas.

Para determinar la incidencia de las diferentes especies de microhongos presentes en el suelo (método de dilución). se utilizó el concepto de abundancia relativa como porcentaje del total de aislamientos, de la siguiente manera.

1 a 6.000 u.f.c. $/ g=$ Esporidico (N) $(1-10 \%)$

7.000 a 32.000 u. f. c. $/ g=$ rigecuerate (W') (11-50\%)

$>33.000$ u.f.c. $/ g=$ Domimante (D) $(>50 \%)$

El cálculo guarda relación al porcentaje máximo de colonias obtenido por un género, (65.000 u.f.c. $/ g=$ 100\%, como fue en el caso de Persicillizm ).

En los pastos la frecuencia de cada especie se calculó en base a la presencia de éstas en el total de las muestras ( $n=18$ ). Cada especie fue contabilizada una sola vez en cada placa ( no imporiando si su presencia era mayor). Ejemplo:

Presencia en 1 a 2 placas $=($ e $)$

En 3 a 9 placas $=$ (Ti)

En 10 a 18 placas $=(\mathbb{D})$

\section{RIRSULTADOS}

La tabla 1, enwmera en órden alfabético los grupos de taxa registrados en ambos habitat, con sus respectivas abundancias relativas.

En las especies aisladas por el método de dilución, el número de propágulos obtenidos en las placas de Petri, presentó rangos desde un mínimo de 1000 a un máximo de 65000 u.f.c. /g. de suelo, lo que sirvió de base a la clasificación de dominancia usada en este trabajo.

Se aislaron un total de 53 géneros, 27 en el suelo y 39 en los pastos, con un número casi similar de especies en ambos sustratos(50 y 48 ). Solo 14 especies $(28,9 \%)$ fueron comunes en ambos sustratos (Tabla 1), representadas

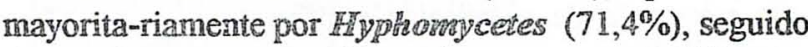
de Coelongucetes $(21,4 \%)$ y Ascomaceres $(7,1 \%)$.

Los taxa exclusivos o mayoritarios en los suelos fueron principalmente especies de: Mucorales, Asperginlus, Ameobasidium, Frasainum, Penicillium y las levaduras blancas y rosadas. Los exclusivos 0 mayoritarios en los pastos fueron: Leptos-

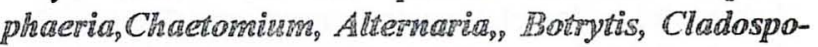
rinum, Dactylasion, Deidsyphian, Ipicoccrm, Heterocoriun, Papralospora, Pericovio, Ramichloridism, Stachyborrys, Tormla y Psendolacinpea..

Los Hipphompeces ya sea hialinos como dematiáceos presentaron las mayores frecuencias de ocurrencia en ambos sustratos, $75 \%$ en los suelos y $78 \%$ en los pastos, mientras de las 9 especies de Coelomaycetes aisladas, 7 corresponden a los pastos y 5 a los suelos. Los Ascomperes fueron poco representados en ambos habitat pero 4 de 5 especies se obtuvieron en los pastos y solo 2 en los suelos.

Cladosporiarse cladorporioides, las especies de Penicillinm y las levaduras blancas, obturvieron las más altas frecuencias relativas en los suelos (>70\%), mientras en los pastos los taxa con la misma frecuencia fueron:

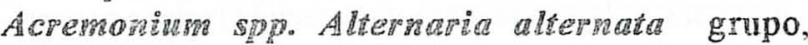

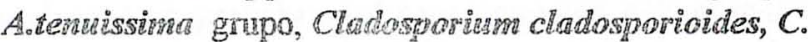

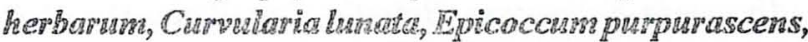

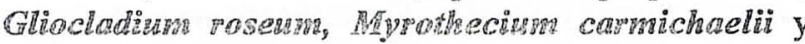
Periconion sprivastissiprer.

A pesar que en los 2 habitat la heterogeneidad de especies es alta, el numero de especies dominantes y frecuentes, fue mayor en los pastos (21 y 13 respectivamente) que en los suelos ( 3 y 6 respectivamente). Estos últimos fueron representados mayoritariamente por una gran diversidad de especies esporádicas (40) y solo 15 con la misma categoría ex los pastos.

\section{MISCUSTON}

Una limitación de nuestra investigación fue abarcar solamente la micota presente en una época 
estacional. Sin embargo, la metodología empleada en su estudio permitió detectar la allta diversidad taxonómica de estos microorganismos que poseen un relativo corto período generacional, así como una gran eficacia en la colonización y explotación de recursos externos, bajo condiciones ambientales fluctuantes. Esto involucra la aplicación de variadas y flexibles modalidades econutricionales (capacidad saprofitica competitiva), en relación a su alta versatilidad y adaptabilidad enzimática (Luttrell, 1974, Cooke \& Whipps, 1987). Esta diversidad es atribuible, en la literatura, a factores tales como: las características intrínsicas de las especies en un habitat determinado; la diversidad de plantas terrestres (Hutchinson, 1959); la proporción de macro y microelementos; la relación $\mathrm{C} / \mathrm{N}$; la presencia de sustancias antifúngicas y los efectos antagónicos o asociados entre los microorganismos presentes en el habitat (Vardavakis, 1990).

Mediante el empleo de datos de presencial ausencia, reunimos a las especiesen 3 categorías ecológicas, acorde con su abundancia relativa, observándose un modelo de distribución francamente diferente entre el habitat del suelo y el de pastos senescentes. Esto confirma la selectividad que habitat-sustrato ejercen sobre ambas comunidades, en especial en la cantidad de especies dominantes en los pastos, situación que en los suelos es reemplazada por un alto número de taxa esporádicos. Esto último, en una comunidad, se atribuye generalmente a una extensiva diferenciación del nicho (Hutchinson, 1959), en especial a nivel de suelo por su alta heterogeneidad de habitat y la presencia de animales de pastoreo que promueven la expansión de especies menores (Christensen, 1989). Varias de las especies que presentaron categoría de dominantes o frecuentes, son consideradas en la literatura como colonizadores saprotrofos primarios y por ende menos selectivas y más adaptadas a nichos inhospitos que los colonizadores secundarios (Frankland, 1975, Bruehl, 1976, Hudson 1986). Estos adquieren gran ventaja en el momento de la senescencia, en especial gracias a su amplia distribución, como es el caso de los géneros Acremonizum, Alternaria, Epicoccum, Botrytis, Curvellarics, Fusarisust, Leptosphaseria y Torulda. Sin embargo, no es fácil poder separar entre éstos a los patógenos o potencialmente patógenos que compiten por el mismo sustrato. Esta apreciación debe ser ratificada por un estudio temporal de nuestra comunidad.

Los pastos analizados, se decomponen y desaparecen rápidamente al integrar la litera vegetal en el tiempo, esta situación les permite soportar un mayor número de especies saprotrofas primarias que las estructuras vegetales que persisten largo tiempo en los suelos. En especial de aquellos taxa capaces de tolerar con mayor eficiencia la desecación, por sus estructuras pigmentadas y de sobrevivencia tales como, esclerocios, microesclerocios y clamidosporas (Hudson 1986). Es el caso de la mayoría delos hongos dematiáceos dominantes $(66,6 \%)$ y frecuentes (61,5\%)aislados en estos vegetale, porcentajes obtenidos del total de hongos pertenecientes a estas categorias. Algunos poseen ciclos de vida a veces tan largos como el habitat colonizado, lo que les permitereproducirse después que los tallos y hojas adquieren senescencia (Thomas \& Shattock, 1986).

La labor de los colonizadores primarios (considerados como epífitos), no solo se efectúa en el filoplano, sino que continúa en abundancia en algunos de los eventos de la senescencia de hojas tallos e inflorescencias, y en la litera vegetal, como se observa en Clcolosaporizm cladosporioides, Abrernaria alternata, Penicillium spp - y Trichoderma harzicanum.

Dentro de las especies que se destacan, Cladosporiums cladosporioides, fue la única común en dominancia en ambos sustratos. Su condición de cosmopolita y su abundante distribución aérea, la convierten en uno de los más efectivos colonizadores primarios prevalentes en diversos tipos de suelos, filoplano y sustratos vegetales senescentes (Dickinson, 1965, Christensen, 1981, Thomas \& Shattock, 1986).

Acremonimm spp., Alternaria alternata, A.tenuissimen, Epicocerm puppurascens y Periconia mirnutissime, mantuvieron la mayor abundancia relativa $(100 \%)$ en todas las muestras de pastos, condición que en los suelos no se presentó, salvo la presencia esporádica de A. teruissima y algunos integrantes del género Acremonium. En los pastos los 2 grupos de Alternaria, mantienen también su condición de cosmopolitas (Ellis, 1971), mientras Lacey (1975) considera $A$. alternata como el integrante más común de las esporas del aire sobre los pastos. Su oportunismo le permite adaptarse a categoríaseconutricionales diversas (saprotrofia, biotrofia), hasta ciertos grados de especialización de algunas cepas (patotipos), capaces de producir micotoxinas hospederoespecificas (Nishimura etal. 1982). Estaparticular habilidad podría permitirle, en nuestro caso, interacciones selectivas hacia otros integrantes de la comunidad fúngica.

La mayoria de las especies de Fusarium se aislaron en forma esporádica desde el suelo, sin embargo F. poae se presentó exclusivamente en los pastos. Su presencia puede atribuirse a la especialización de esta especie en estos vegetales, representados en nuestro trabajo principalmente por integrantes de la familia Poaceae de las gramíneas. Su poder patógenoes reconocido en los pastos cultivados (Smith et al. 1989), mientras la escasa selectividad de $\mathbb{F}$. oxisporium, le permite atacar una gran diversidad de hospedadores ya sea vegetales o animales, corno así de subsistir en muchos ambientes telúricos. Tollenaar et al (1970) detectan en Chile sobre Dactylis glopperato la presencia de $\mathbb{F}$. roseum fr. cerealis

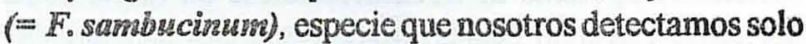
en los suelos. 
Tabla 1.

Abundancia relativa de taxa aislados (suelo y pastos) según categoría y método

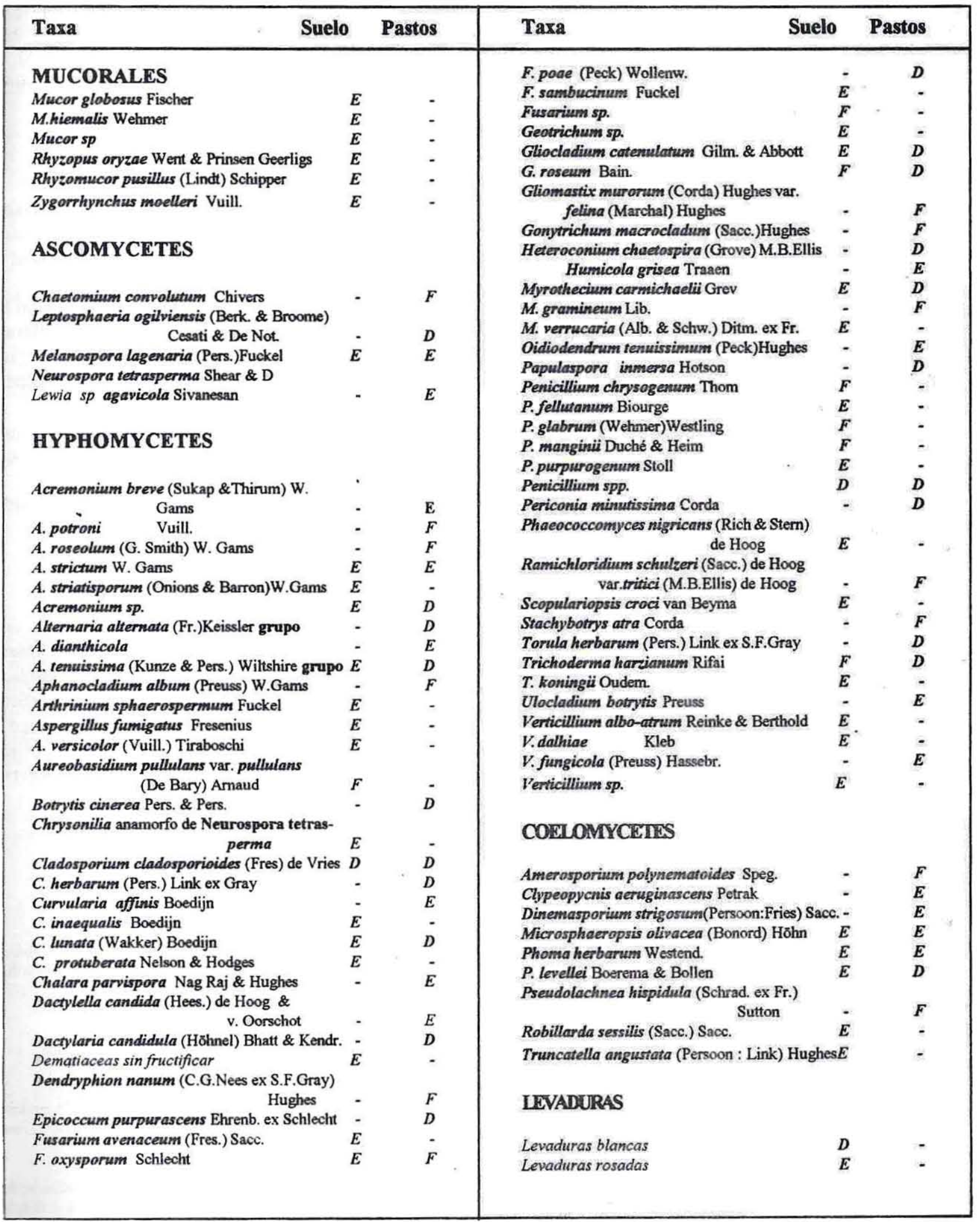


Botrytis cinerea, no es considerado como saprofito en la comunidad de especies graminícolas (Lacey, 1975)., sin embargo su abundancia puede asociarse a la alta humedad reinante en las placas de Petri. Su crecimiento no fue aparentemente afectado in "vitro" por la capacidad antagonistica de Trichodersmes learricosum, compartiendo ambos en dominaricia el mismo substrato. La misma situación aconteció con el antagonismo deE.pusponsascens, frente a algunas especies de Alrepnoside (Pace \& Campbell, 1974).

Pensábamos encontrar una mayor cantidad de Coelonycetes, pero sólo Amerosporivan polynematoides, Phoma levellei y Pseudolachnea hispidulda, obtuvieron las mayores frecuencias relativas en los pastos. De éstos sólo $\mathbb{P}$. levellei se presentó esporádicamente también en el suelo, posiblemente debido a la aparición tardía de estos taxa en los pastos senescentes, pueden consi-derarse como colonizadores secundarios.

$\mathrm{La}$ ausencia de levaduras que normalmente cumplen sus ciclos de vida en el filoplano (Sporobolomycetaceas y Cryptococcacere), seguramente fue afectada por la metodología empleada o las condiciones adversas ambientales sobre el sustrato senescente.

Queremos destacar algunas de los taxa poco comunes que hemos aislado, tales como Scopularriopsis croci, Lewìiasp. (con anamorfo enAltermaria), Verticillium fungicola y Dactylella candi-des. Esta última especie fué primeramente determinada como Dactyiaria candides (Nees:Fr.) Sacc. , (= Dactylium candidam Nees, = Candelabrella candida (Nees:Fr.) Rifai, = Arthrobotrys candidas (Nees:Fr.) Schenck, Kendrick \& Palmer), sin embargo, segun De Hoog \& v.Oorschot (1985), es mejor clasificada como Daciylella carndida. Es considerada como un predador de Nemátodos, que presenta anillos no retráctiles y prominencias piriformes que se adyeren a estos invertebrados (Dowsett \& Reid 1977), estructuras que hemos observado directamente sobre el sustrato herbáceo colonizado.

Nuestro listado de especies, ciertamente no revela las complicadas interacciones bióticas, ni las caracterizaciones por función de estas especies, presentes en el ecosistema, sin embargo permite conocer en parte su distribución biogeográfica y la estructura de su comunidad en relación al ambiente, situación poco considerada a nivel nacional en los estudios sinecológicos de la cobertura vegetal de pradera.

\section{CONCLUSIONES}

La mayor diversidad de especies se observó en ambos habitat para los integrantes de los HHpphomycetes, particularmente dominantes en áreas foliares, tallos e inflorescencias de pastos senescentes.

Las mayores diferencias entre ambos habitat se observaron en los grupos deMincorcles y las Levaduras, los cuales no se presentaron en los pastos y para Asconycetes y Coelomycetes, que aparecieron principalmente en estos últimos.

Se aislaron Coelonaycetes, aunque con bajas abundancias relativas. Estos sólo dominaron en pastos, especialmente Phoma levelle:

La mayoría de las especies detectadas no han sido reportadas en la literatura nacional en éstos 2 habitat.

\section{AGRADECIMUINTOS}

Se agradecela cooperación del Prof. Otto Z⿱öliner, del Departamento de Botánica de la Universidad Católica de Valparaiso, en la determinación de los pastos y a la Sra. Ornella Piontelli en la ayuda prestada en el muestreo.

Quiero agradecer en forma especial, los útiles comentariosy sugerencias, efectuadas por el Dr. J.Guarmo, en la revisión de este manuscrito.

\section{RRITIRENCIAS}

Bon, M. \& Gehu, J. M. (1973) Unites superieures de vegetation et racoltes mycologiques.Doc.Mycol. $6: 1-40$

Bruehel, G. W. (1976) Management of food resources by fungal colonists of cultivated soils. Ann. Rev. Phytopathol. $14: 247$ 264

Clarke, D. C. \& Christernser, M. (1981) The soil microfungal community of a south Dakota grassland. Can. J., Bot. 59: $1950-1960$

Cooke, R. C. \& Whipps, (1987) Saprotophy, stress and symbiosis. In: Evolutionary biology of the fungi. Eds. Rainer, A.D. M., Brasier, C.M. \& Moore, D. Cambridge University Press pp. $137-148$
Clnristensen, M. (1981) Species diversity and dominance in fungal communities. In : The fungal community, its organization and role in the ecosystem. Eds. Wiclow, D. T. \& Carroll, G.C.Marcel Dekker, Inc., N.York. pp. 201-232

Christensem, M. (1989) A view of fungal ecology. Mycologia 81 : $1-19$

Dichineson, C. H. (1965) The mycoflora associated with Halimione portuliacoides. III. Fungi on green and moribund leaves. Trans. Br. mycol. Soc. 48 : 603-610

Dowsett, J.A. \& Reidy. (1977) Light microscope observations on the trapping of nematode by Dactylaria camelids. Can. J. Bot. 55 :2956-2962 

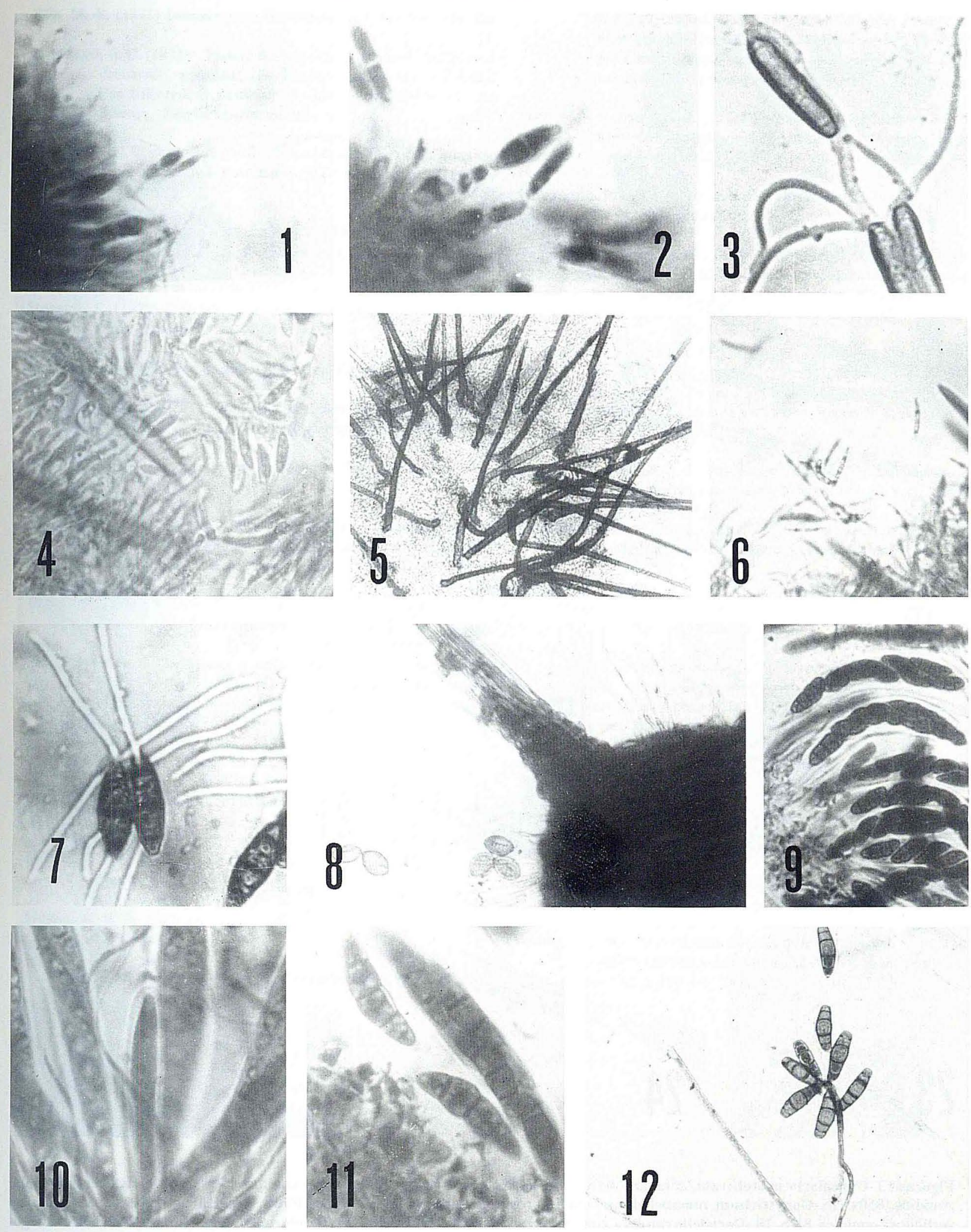

Figuras.1,2,3. Robillarda sessilis. 1.- Conidios y apéndices en la zona basal del conidioma, 850x. 2.- Células conidiógenas, desarrollo conidial y apéndices apicales, $1000 \mathrm{x}$. 3.- Conidios y apéndices, 1000x. 4.- Dinemasporium strigosum, setas, células conidiógenas y conidios con apéndices, $1000 \mathrm{x}$. 5,6.- Pseudolachnea hispidula. 5.- Conidioma, setas y conidios, 200x. 6.- Conidios y setas, 500x. 7.-Truncatella angustata, conidios y apéndices, $850 \mathrm{x}$. 8.- Melanospora lagenaria, ascoma y ascosporas, 200x .9.-Lewia sp, ascos y ascosporas muriformes, 400x. 10,11.Leptosphaeria ogilviensis. 10.- Hamatecium, ascos y ascosporas inmaduras, 850x. 11.- Ascosporas maduras, 850x. 12.-Curvularia inaequalis, conidios y conidióforos, $500 \mathrm{x}$. 


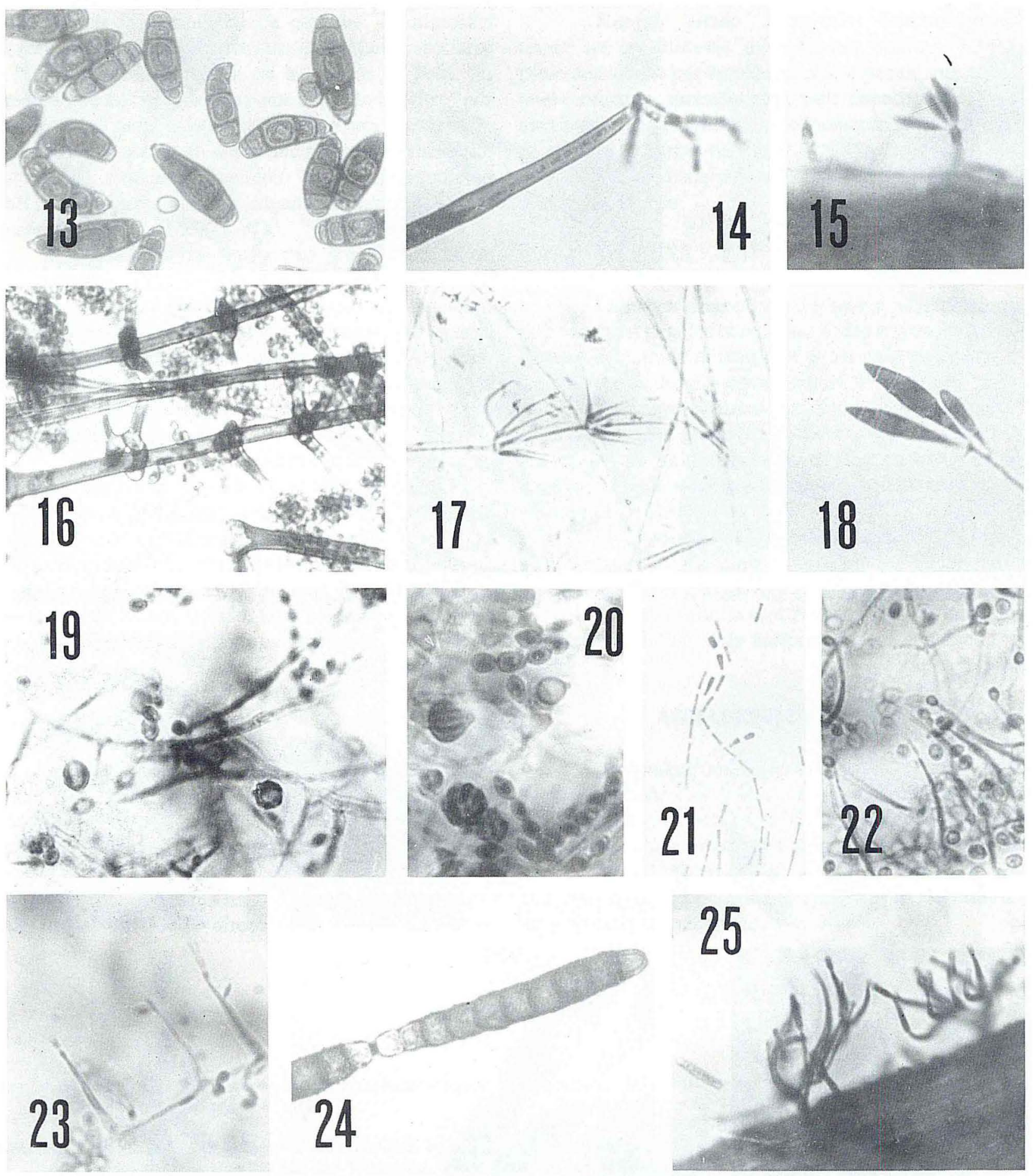

Figuras.13.-Curvularia protuberata, conidios, 400x. 14.-Chalara parvispora, conidióforo y conidios, 500x. 15.-Dactylaria candidula, conidios, 850x. 16.-Gonytrichum macrocladum, conidióforos y conidios, 500x. 17.-Verti-cillium fungicola, células conidiógenas en verticilos y conidios, 850 x. 18.-Dactylella candida, conidios y conidióforos, 500x. 19,20.-Acremonium striatisporum, 19.-Células conidiógenas y conidios, $850 \mathrm{x}$. 20.-Conidios estria- dos, 1000x. 21.-Geotrichum sp, artroconidios, 450x. 22.-Acremonium breve, células conidiógenas y conidios, $850 x$. 23.-Acremonium roseolum, células conidiógenas y conidios, $850 x$. 24.-Dendryphion nanum, conidios en cadena, $500 x$. 25 .Heteroconium chaetospira, células conidiógenas y conidios sobre sustrato herbáceo, $500 \mathrm{x}$. 
Ellis, M. B. (1971) Dematiaceous Hyphomycetes.C.M.I. Kew England

Franklanal, J. C. (1975) Fungal decomposition of leaf litter in a deciduous woodland. In: Biodegradation and humification Eds.Kilbertus, G.,Reisinger, O., Mourey, A. \& Cancela da Fonseca. Pierron Sarreguemines. pp. 33-40

Hass,H.(1932) Die bodenbewohënden grosspilze in den waldfornation eininger gebeide von württenburg. Beih.Bot.Centralbl. Abt.B. 50: $35-134$

Hoog, G. S \& Oorschot, C.A.N. van. (1985) Taxonomy of Dactylariฎ complex VI. Key to the genera and check-list of epithets. Studies in Mycol. $26: 97-122$

Hubalek, Z. (1978) Coincidence of fungal community associated with birds. Ecology $59: 438-442$

Hudson, H. J. (1986) Fungal biology. Edward Amold. London.

Hutchinson, G. E. (1959) Homage to Santa Rosalia or why are there so many kinds of animals?. Amer. Naturalist $93: 145-159$

Lacey, J. (1975) Airborne spores in pastures. Trans. Br. mycol, Soc. $64: 201-208$

Lamb, R. J. \& Brown, J.F. (1970) Non-parasitic microflora on leaf surfaces of Paspuluam dillatatum, Salix babylonica and Eucaliptuss stellulata. Trans. Br. mycol. Soc. 55: 383-390

Lange, M. (1978) Fungus flora in August, ten years observations in a danish beach wood district. Bot. Tids-skrift. $73: 21-54$

Leischner-Siska, E. (1939) Zur soziologie und oekologie der höheren pilze. Untersuchung der pilzvegetation in der umgebung von Salzuburg während des maximalaspektes 1937. Beih. Bot. Centralblatt 59: $359-429$

Losa Quintana, J. M., Romero Rodriguez, C. \&t García Perez, J.C. (1980) Aportación al estudio micosociológico de la provincia de Leon. Acta Bot. Malacintana 6: 75-82.

Luttrell, (1974) Parasitism of fungi on vascular plants. Mycologia 66:115

Mujica, R. F. \& Vergara, C. C. (1980) Flora fungosa Chilena. Ed Universitarias .Santiago

Nishimura, S.,Kohmoto, K., Otani, HI., Ramachandran, P. \& Tamura,
F. (1982) Pathological and epidemiological aspects of A. silteranats infection depending on a host speciftoxin. In: Plant infection: the physiological and biochemical basis. Jap. Sci. Soc. Press, Tokio Springer/ Verlag, Berlin. pp.199-214

Prce, M.A. of Crnpbelip, $R$ (1974) The effect of saprophytes on infections of leaves of Brassica spp. by Alicermaria brassicae. Tran. Brit mycol Soc. 63 : 193-196

Quinntanillla, P.V. (1983) Geografia de Chile. Tomo III. Biogeografia. Inst Geográfico Militar.Santiago

Rambelli, A, Persiani, A. M., Maggi, O., Onofri, S., Riess, G., Dowgiallo, G. \& Puppin,G. (1983) Comparative studies on microfungi in tropical ecosistem. Mycological studies in South Western Ivory Coast forest. Report $N^{\circ} 1$, Mab-Unesco. Roma

Rambelli, A., Persinni, A. M., Maggi, O., Onofri, S., Riess, G., Dowgiallo, G. \& Zucconi, L. (1984) Comparative studies on microfungi in tropical ecosistem. Mycological studies in South Western Ivory Coast forest. Report $N^{\circ} 2$, Girn. Bot. Ital. $118: 201-243$

Smith, J. D., Jacksen, N. \& Woolhouse, A. R. (1989) Fungal diseases of amenity turf grasses. E and F.N.Spon Ltd. N. York

Thomas, M. R. \& Shattocha, R. C. (1986) Filamentous fungal association in the phylloplane of Lolinum peremine. Trans. Br. mycol Soc. $87: 255-268$

Tollenaar, et al. (1970) Observacion de nuevas enfermedades vegetales en Chile. Agr. Tec. Chile 30:51-54

Vardayalds, E. (1990) Seasonal fluctuations of soil microfungi in correlation with some soil enzyme activities and VA mycorrhizae associated with certain plants of a typical caicixeroll soil in Grece. Mycologia 82 : 715-726

Warkap,J.H. (1950) The soil plate method for isolation of fungi from soil. Nature 166:117-118

Warkup, J.H. (1951) The ecology of soil fungi. Trans. Brit. mycol. Soc. $34: 376-399$

Wicklow, D.T. (1973) Microfungal population in surface soils of manipulated prairie stands. Ecology 54 : 1302-1310

Widden, P. (1986) Microfungal community structure from forest soils in southern Quebec, using discriminant function and factor analysis. Can J. Bol. 64 : 1402-1412 